\title{
Magnetic field induced random pulse trains of magnetic and acoustic noises in martensitic single-crystal $\mathrm{Ni}_{2} \mathrm{MnGa}$
}

\author{
Lajos Daróczi, Eszter Piros, László Z. Tóth, and Dezső L. Beke \\ Department of Solid State Physics, University of Debrecen, H-4002 Debrecen, P.O.Box 400, Hungary
}

(Received 22 November 2016; revised manuscript received 17 March 2017; published 13 July 2017)

\begin{abstract}
Jerky magnetic and acoustic noises were evoked in a single variant martensitic $\mathrm{Ni}_{2} \mathrm{MnGa}$ single crystal (produced by uniaxial compression) by application of an external magnetic field along the hard magnetization direction. It is shown that after reaching the detwinning threshold, spontaneous reorientation of martensite variants (twins) leads not only to acoustic emission but magnetic two-directional noises as well. At small magnetic fields, below the above threshold, unidirectional magnetic emission is also observed and attributed to a Barkhausen-type noise due to magnetic domain wall motions during magnetization along the hard direction. After the above first run, in cycles of decreasing and increasing magnetic field, at low-field values, weak, unidirectional Barkhausen noise is detected and attributed to the discontinuous motion of domain walls during magnetization along the easy magnetization direction. The magnetic noise is also measured by constraining the sample in the same initial variant state along the hard direction and, after the unidirectional noise (as obtained also in the first run), a two-directional noise package is developed and it is attributed to domain rotations. From the statistical analysis of the above noises, the critical exponents, characterizing the power-law behavior, are calculated and compared with each other and with the literature data. Time correlations within the magnetic as well as acoustic signals lead to a common scaled power function (with $\beta=-1.25$ exponent) for both types of signals.
\end{abstract}

DOI: 10.1103/PhysRevB.96.014416

\section{INTRODUCTION}

The magnetic shape memory effect (MSE) is based on the magnetic field induced reorganization of martensitic variants in ferromagnetic shape memory alloys since this detwinning process results in large macroscopic deformations [1-8]. This fast and large superplastic deformation can be used in different applications such as in shape morphing or actuators $[1,3,4]$. In general, the multivariant martensitic structure, which is formed during cooling of the austenite, can be transformed into a single variant one by application of either uniaxial stress or magnetic field. It was shown in Ref. [9] that acoustic emission (AE) activity can be detected during stress-induced martensite reorientation, indicating the jerky character of the detwinning process. It is plausibly expected that during martensite variant rearrangements, due to the strong magnetoelastic coupling, rearrangements of the magnetic domains take place as well. Indeed, in Refs. [10,11], magnetic noises during strain induced detwinning were detected. This intermittent character is similar to the behavior of the magnetic field induced classical Barkhausen noise $(\mathrm{BN})$, which is due to the intermittent rearrangement of the magnetic domain structure by the motion of domain walls (magnetic avalanches) [12].

Recently, investigations of systems responding to slow external driving by exhibiting scale-free avalanche dynamics or crackling noise received increased interest [13-16]. Indeed, it was shown that $\mathrm{AE}$ can be evoked, for example, by austenite/martensite phase transformations [17-20] and plastic deformations $[10,11,21,22]$ too. The above noises, indicating the behavior near a critical point, can be characterized by powerlike distribution functions [17,23],

$$
P(x)=C x^{-\beta} \exp \left(-x / x_{c}\right),
$$

where $C$ is the normalization constant, $P(x)$ is the probability density of the given parameter $x$ (the amplitude, area, energy or duration of the individual peaks of the noise), $\beta$ is the critical exponent, and $x_{c}$ is the cutoff value.

For better understanding of the details of the noisy character of the magnetic field induced detwinning process, it is important to determine the statistical characteristics of acoustic and magnetic noses (similarly as it was done in Refs. [10,11] for magnetic noises obtained during strain induced detwinning) and to investigate how these change with changing magnetic field. In Ref. [9], no statistical analysis of the stress induced AE noises was presented and only a qualitative description was given. Furthermore, in previous studies of magnetic field induced detwinning, no noise measurements were carried out, but rather the emphasis was put on the observation of the initial and/or final magnetic domain structure [24-29]. In Ref. [29], a detailed study of twin boundary motion, over a wide range of magnetic fields, together with in situ magnetic domain observations was presented. It was concluded that no magnetic domain wall motion during twin boundary shifts took place. This was a bit different from the magnetomicrostructural mechanism proposed earlier in Ref. [30], in which both domain wall motions and rotations are included (see also below).

Thus our primary interest was to determine the characteristic exponents for AE jerks emitted during magnetic field induced superplastic deformation in single-crystalline $\mathrm{Ni}_{2} \mathrm{MnGa}$ ferromagnetic shape memory alloys with $10 \mathrm{M}$ modulated structure. Furthermore, simultaneously with the AE noise, we also detected magnetic emission (ME) signals during the same process. This will make possible the comparison of the critical exponents of ME and AE like it was done for the noises emitted during austenite/martensite phase transformation in the same alloy [18]. Comparing the critical exponents of ME signals obtained from periodic bending deformation of martensitic $\mathrm{Ni}_{2} \mathrm{MnGa}$ samples $[10,11]$ and from magnetic field induced detwinning, we can check whether the critical exponents of the ME noises are the same for the two different modes 
of excitations (strain or magnetic field induced detwinning). Furthermore, the critical exponents of classical Barkhausen noise, obtained from magnetic domain rearrangements during magnetization along hard and easy magnetization directions will also be compared with exponents of the two universality classes [31-33] known in the literature. On the basis of our results, we will also analyze temporal correlations within the acoustic and magnetic signals measured.

\section{EXPERIMENTAL}

The effect of a magnetic field (up to $0.95 \mathrm{~T}$ ) on the twin structure is investigated in $\mathrm{Ni}_{50} \mathrm{Mn}_{28.5} \mathrm{Ga}_{21.5}$ singlecrystalline alloy with a $10-\mathrm{M}$ modulated martensitic structure $\left(1 \times 2.5 \times 20 \mathrm{~mm}^{3}\right.$, purchased from Adaptamat Co., Finland). All the deformation, acoustic emission, and magnetic noise measurements are performed at room temperature, where the sample is in a fully martensitic state. The field rate during the measurements is $4 \times 10^{-3} \mathrm{~T} \mathrm{~s}^{-1}$. Deformation measurements are done by a Mitutoyo $\mathrm{C} 112 \mathrm{~A}$ strain gauge. The collection of magnetic and acoustic emission signals is implemented by using a National Instruments DAQ PCI 6111 data acquisition device. The acoustic signals are detected by a MICRO80 piezoelectric sensor produced by Physical Acoustic Corp. (For the details of the noise detections and data collection, see also Refs. [10,11,18].)

The experimental setup is shown in Fig. 1. The sample (1) is fitted in a plastic sample holder. The magnetic detector coil (2) is wounded directly on the sample (320 windings). The lower end of the sample is connected to the piezoelectric acoustic sensor (3), using silicon grease as coupling media. The upper end of the sample is connected to a strain gauge (4) via a supplementary plastic rod (5). The measuring force (spring force of the gauge plus the weight of the supplementary rod) is about $0.9 \mathrm{~N}$. The sample is placed into the air gap of a Weiss-type electromagnet (6). The direction of the applied

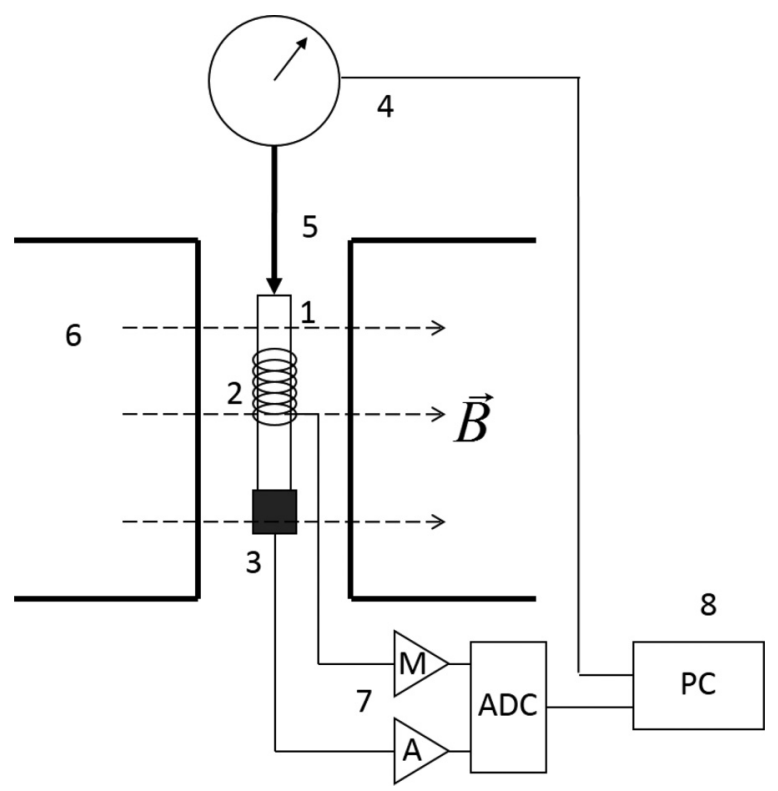

FIG. 1. Experimental setup, schematically ( $\mathrm{M}$ and A refer to magnetic and acoustic signals respectively; see also the text). magnetic field is perpendicular to the longitudinal axis of the sample, which is along the [100] direction of the austenite phase. The actual magnetic field in the air gap is calculated from the linear change of the excitation current taking into account the saturation of the iron core at higher fields by using a calibration curve.

In order to have a well-defined initial state, the sample is pretreated as follows: (i) we increase the external magnetic field to reach the maximum strain, (ii) we remove the magnetic field, and (iii) we compress the sample to create a welldefined (single variant) martensitic state. The acoustic and magnetic emission signals during the change of the magnetic field are detected simultaneously by the amplifier (7) and the acquisition unit. We used home-made 60-dB amplifiers, with bandwidths of $0-200 \mathrm{KHz}$ and $0-2 \mathrm{MHz}$ frequency range for the magnetic and acoustic signals, respectively. A direct-current coupled amplifier is used, so the low-frequency range of the signal is not filtered. The advantage of the ac coupled amplifiers is the stable baseline and the immunity from low-frequency external noises. On the other hand, the ac coupling makes an inevitable distortion of the pulse shape (all positive peaks are followed by a negative pulse because of the coupling capacitor). The dc coupling mostly eliminates the above problem but needs careful shielding of the external electric and magnetic fields and good temperature stability of the amplifiers. For acoustic emission signals, this is not a cardinal problem because of the capacitive nature of the piezoelectric sensors (the sensor itself is a high-pass filter), but in case of inductive magnetic sensors, the dc coupling in some cases can be useful, especially for investigation of pulse shapes. The sampling rate of the analog-to-digital converter (ADC) is 5 Msample/s. The signals are collected on a PC (8) and the statistical data are evaluated off-line. These measurement settings are based on our many years of experience in magnetic noise measurements [34,35].

\section{RESULTS}

The simultaneously detected magnetic and acoustic noises and the corresponding elongation data $\left(\Delta L / L_{0}\right)$ are shown in Fig. 2 as a function of the increasing magnetic field. The initial state-as it was mentioned above-is produced by compression. In the low-field range $(0-0.30 \mathrm{~T})$, very intensive unidirectional magnetic signals are detected. In the high field range $(0.35-0.95 \mathrm{~T})$, sporadic magnetic noise (with mostly unidirectional peaks), together with intensive acoustic activity, are present. A macroscopic shape change of about $6 \%$ takes also place in this high-field range.

According to Fig. 3, the high field acoustic and magnetic emission pulses show strong correlations: the simultaneously recorded signals, plotted in the $0.72-0.75 \mathrm{~T}$ field range, illustrate it quite well. The insert shows, as an enlarged part of the main figure, the shapes of the acoustic and magnetic signals (after cutting off the background noise levels: about $20 \mathrm{mV}$ for both magnetic and acoustic signals). The classical threshold-based method is used for the identification of the magnetic avalanches and the acoustic wave packets. The magnetic avalanche starts when the absolute value of the signal is higher than the threshold level and finishes when it goes under the threshold. The AE events consist of oscillating peaks 


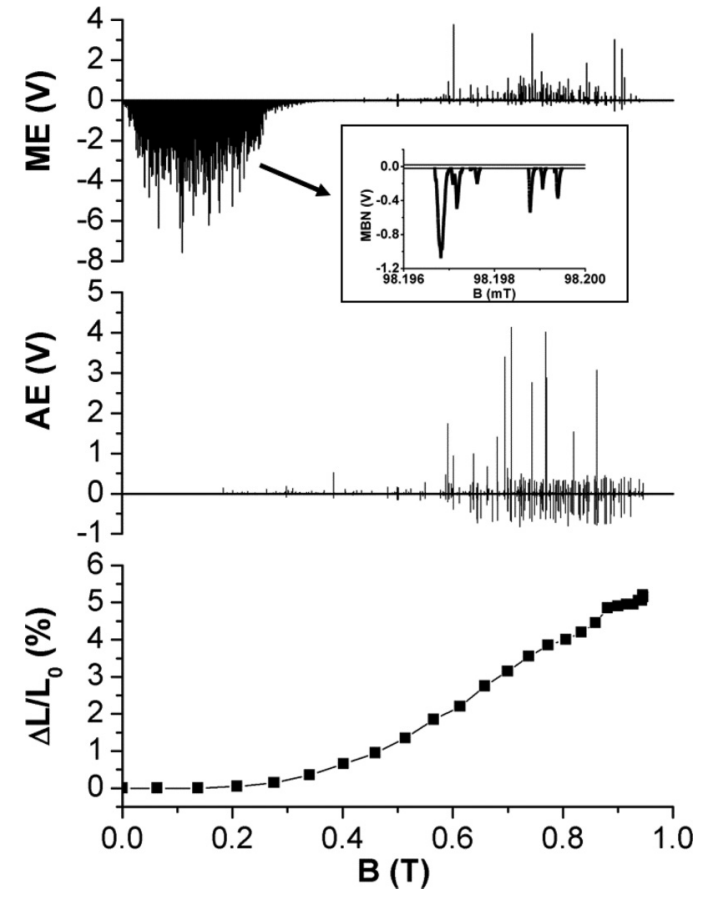

FIG. 2. Magnetic emission, ME, acoustic emission, AE, and relative deformation, $\Delta L / L_{0}$, as a function of increasing magnetic field. The insert shows the shapes of the magnetic signals in the enlarged part of the unidirectional noise package.

with decreasing amplitude. Thus we use the usual definition: the acoustic event starts when the first oscillation crosses the threshold and finishes when the amplitude of the oscillation falls below the threshold. The peak amplitude is the maximum

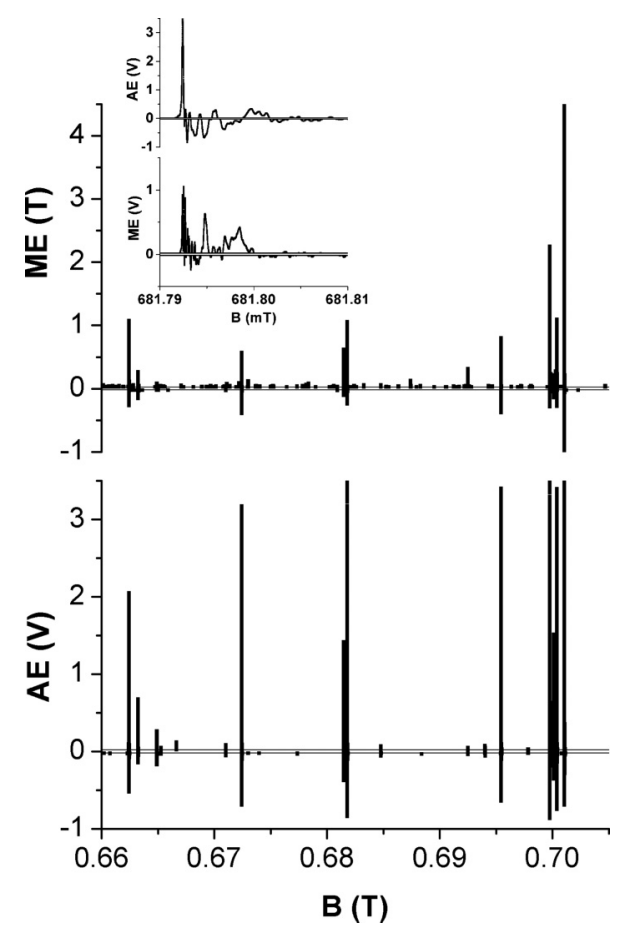

FIG. 3. Coincidence between magnetic and acoustic emission peaks (ME and $\mathrm{AE}$, respectively). The insert shows the shapes of two coinciding MA and AE signals: see also the text.

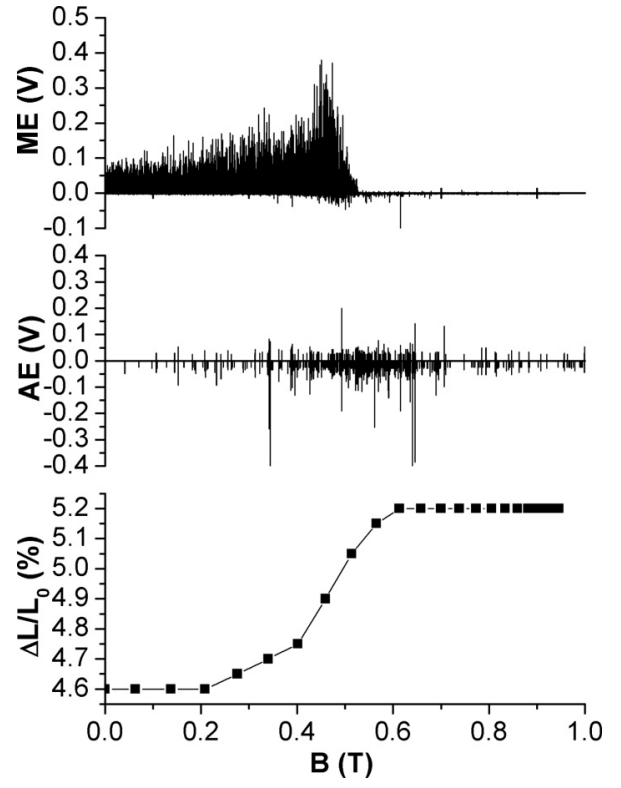

FIG. 4. Magnetic emission, ME, acoustic emission, AE, and relative deformation, as a function of decreasing magnetic field in the second cycle.

absolute value of the voltage signal between the start and finish of the event. The energy of the peak is calculated by using the

$$
E_{i}=\frac{1}{R} \int_{\text {start }_{i}}^{\text {finish }_{i}} V^{2}(t) d t
$$

expression, where $V^{2}(t)$ is the square of the measured signal, and $R$ is an arbitrary chosen resistance, $1 \mathrm{M} \Omega$.

Signals as a function of the decreasing magnetic field are shown in Fig. 4. Weak unidirectional magnetic noise (compare the units on the vertical axes in Figs. 2 and 4) in the low-field range and low-level acoustic activity are observed between 0.35 and $0.60 \mathrm{~T}$, where a very small macroscopic shape change (about $0.5 \%$ contraction) is measured too.

Figure 5 shows the acoustic and magnetic signals and the elongation as the function of increasing magnetic field (second cycle). Low-level unidirectional ME noise and negligibly small $\mathrm{AE}$ activity is detected at low-field values. In the high-field range, a small elongation is observed with the same magnitude as the contraction on Fig. 4 (but at field larger by about $0.3 \mathrm{~T}$ ). In addition few two-directional ME (i.e., peaks both with up and down voltage signals) and AE peaks can be seen in the $0.8-0.95 \mathrm{~T}$ interval.

Figure 6 shows the magnetization curves during the first (open symbols) and second cycle (full dots). The first run started from zero field value (along the hard magnetization axis) and the arrow indicates the beginning of detwinning. After saturation, the magnetization decreases with decreasing field along the curve given by full dots and follows this curve during further cycling between -1.2 and $+1.2 \mathrm{~T}$. This curve is very similar to the magnetization curve obtained from a similar measurement and shown in Fig. 9 of Ref. [27]. It can be seen that the slopes of the curves (at around $B=0 \mathrm{~T}$ ) belonging to magnetization along hard and easy directions, as expected, are characteristically different. 


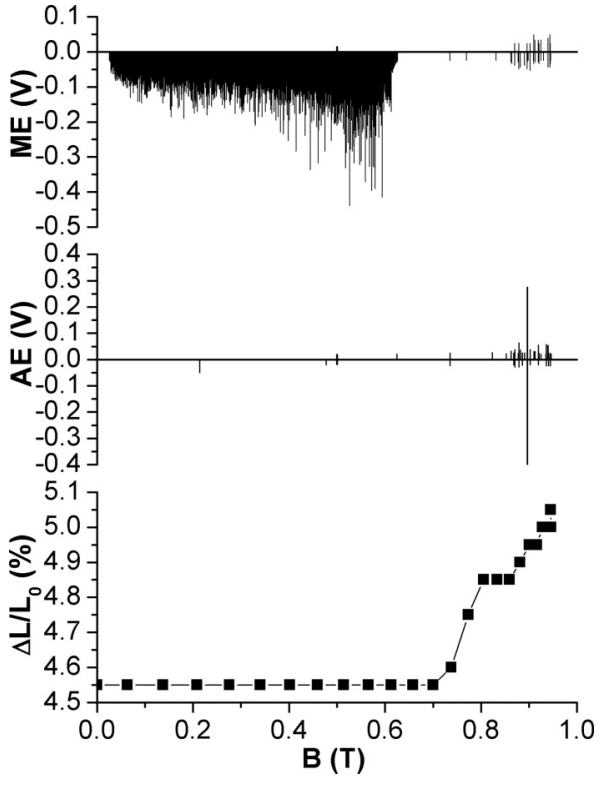

FIG. 5. Magnetic emission, ME, acoustic emission, AE and relative deformation $\Delta L / L_{0}$, as a function of increasing magnetic field in the second cycle.

\section{DISCUSSION}

\section{A. Magnetomicrostructural mechanism of twin and magnetic domain rearrangements}

Our results on the field dependence of the relative deformation, $\mathrm{AE}$ and $\mathrm{ME}$ noises during the first run show that at a certain crossover value of $B$ the magnetic field induced detwinning started (compare Figs. 2 and 6). Below this field value, the magnetization took place along the hard magnetization direction. During the second cycle (i.e., after changing the variant structure, indicated by the elongation) magnetization took place along the easy magnetization direction. Thus for the interpretation of $\mathrm{ME}$ and $\mathrm{AE}$ noises measured, we have to use an appropriate magnetomicrostructural model in accordance with the above observations.

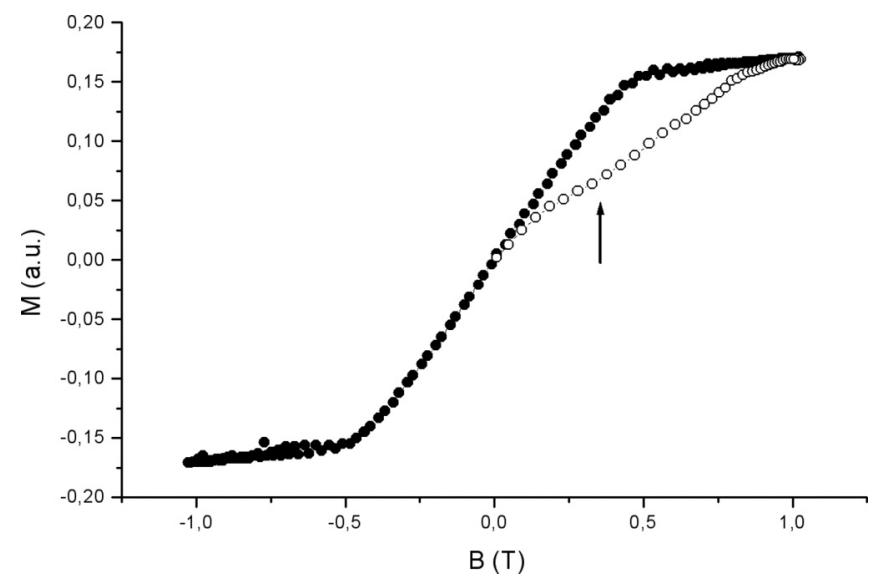

FIG. 6. Magnetization curves during the first (open symbols) and the following cycles (full dots). The arrow shows the beginning of detwinning (see also Fig. 2).
On the basis of the literature results on the changes of the twin and magnetic domain structure during martensite variant rearrangements [24-30], the main features of the magnetomicrostructural mechanism can be summarized as follows (Ref. [30]). (a) In a single variant initial state (produced, e.g., by application of compressive uniaxial stress), the magnetic domains are aligned along the easy magnetization axis ( $c$ axis), separated by $180^{\circ}$ domain walls. (b) When a magnetic field perpendicular to the $c$ axis is applied first, both domain wall motion and magnetization rotation can occur (since a relatively low magnetic field is required for domain rearrangements as compared to variant reorientation/nucleation). (c) At a certain field value, a new variant, with domains aligned parallel with the magnetic field, nucleates. (d) At the same time-together with the increase of the volume fraction of the second variant by the motion of the twin boundaries - magnetization rotation in the first variant, and concurrent domain wall motion(s), occur. (e) Finally, with further increase of the magnetic field, the magnetization in the original variant rotates further and magnetic saturation sets up. This final state can have an incomplete reorientation arrangement, i.e., it can contain martensite variants remained from the initial state.

In addition, direct observations in Ref. [29] revealed that during the (b)-(d) steps no domain wall motion occurred. Furthermore, here the final state had a single domain structure. After the removal of the magnetic field, the sample was in a high remanent state, with a small number of domain boundaries, and the regular domain structure [very similar to the case (a) but rotated by $90^{\circ}$ ] appeared only after demagnetization. This observation is somehow in contradiction with the change of the magnetization during such a magnetic field induced strain (see Fig. 6 and Figs. 4 and 9 in Ref. [27]), where no such high remanent magnetic state was observed. Nevertheless, apart from the above small details, the main features of the mechanism illustrated in Figs. 7 and 8 are well established and we will rely on this in the following discussion.

\section{B. Results on magnetic domain rearrangements}

Increasing the uniaxial magnetic field (Fig. 2) first, before the field reached the switching field necessary for the nucleation of new twin(s) and to start the deformation, unidirectional magnetic signals were detected. This should correspond to classical $\mathrm{BN}$; the increase of the magnetic field brings about random bursts of magnetization leading to a series of unidirectional voltage signals in the detector coil. Different mechanisms can contribute to the noise here, related to domain wall motions and magnetization rotations [27,29,30]. The classical Barkhausen noise is usually interpreted as the result of the intermittent motion of domain walls (pinned at different pinning points) around the coercive field (where the permeability is maximal). However, as was pointed out in Refs. [12,35,36], intensive magnetic noise, with different critical exponents [35] can also be detected at the knees of the magnetization curves at which domain rotations are also observed.

In order to check that the above noise package is indeed the result of pure magnetic domain rearrangements we carried out the following measurements. We stop the increase of the magnetic field at about $0.25 \mathrm{~T}$ (see Fig. 2) before the detwinning process started (and the magnetic noise package 


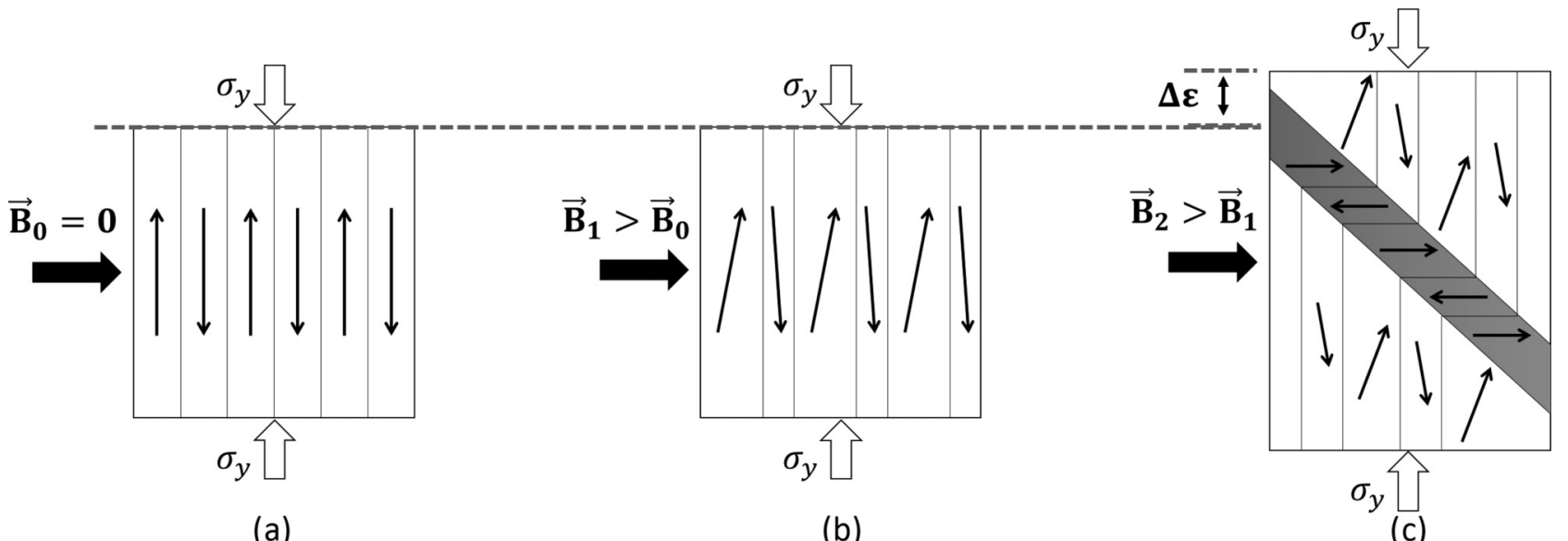

(a)

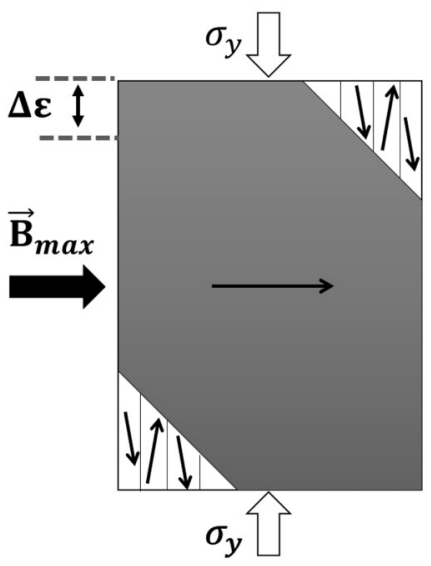

(d)

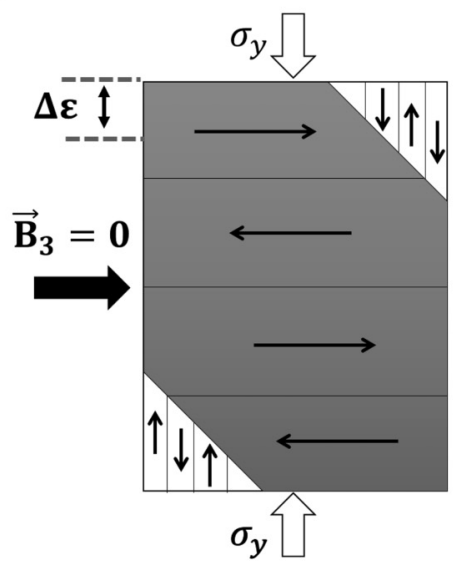

(e)

FIG. 7. Change of the twin and magnetic domain structure (see also Ref. [30]) with increasing external magnetic field $B$ under a small compression due to the measuring force $(0.9 \mathrm{~N} \sim 0.4 \mathrm{MPa})$ : (a) single variant structure (produced by compression), the easy axis of magnetization (c axis) is vertical, the magnetic domains are aligned along the easy axis and separated by $180^{\circ}$ domain walls if $B=0$, (b) with increasing $B$ there are rotations inside the magnetic domains and domain wall motions, (c) at a certain $B$ value new twin nucleates, with $\mathrm{c}$ axis along the field, and thus within this the magnetization is parallel with $B$, (d) further increase of $B$ leads to the increase of the volume fraction of the new twin, during which there are sudden slips in the magnetization as the twin boundary experiences intermittent slips, (e) effect of decreasing the field to zero: one arrives at a variant structure, with a magnetic domain structure similar to (a) but rotated by $90^{\circ}$ (and containing a small fraction of a second variant, remained). Starting from (e) and cycling the field (increasing/decreasing the field), the system switches between (e) and (d).

has been practically finished) and decreased the magnetic field. The results are shown in Fig. 9. It can be seen that as it is expected for a typical Barkhausen noise the reversal of the direction of the magnetic field resulted in unidirectional magnetic noise package with opposite peak directions. In addition to this, we repeated our first run under constrain against detwinning elongation and thus took the full magnetization cycle along the hard magnetization direction (between points $\mathrm{A}$ and $\mathrm{C}$ in Fig. 8). The obtained results are shown in Fig. 10. First, it can be seen that at low-field values we got back the unidirectional noise package shown in Fig. 2 . Second, this figure illustrates that the sample was not fully magnetized before the detwinnig started, and increasing the magnetic field (but hardly constraining the sample in the same variant state) a two-directional noise package developed, too.

In our sample, the initial magnetic domain structure and its changes are different from the usual magnetization experiments (sweeping the magnetic state from $-M_{s}$ to $+M_{s}$ ).
Since we investigated the magnetic field induced deformations in single variant single crystal, we chose the initial state as shown in Fig. 7(a). Let us consider the possible jumps of magnetization starting from this state. During magnetization rotations in part (b) of Fig. 7 the rotation of magnetization in the neighboring domains is such that its horizontal components increase along the applied field, while the sign of the change of its vertical components (this is what we can detect in our sensor coil: see the arrangement in Fig. 1) is opposite and should not result in unidirectional peaks as observed. Furthermore, if the magnetization directions in neighboring domains in Fig. 7(b) have randomly different misalignments (i.e., there are both up and down magnetizations with larger misalignment angles than the others) then the energetically favorable domain wall shifts should have randomly different directions, leading again to peaks with both up and down directions.

For the understanding of the presence of unidircetional magnetic signals let us assume that there is a small deviation, 


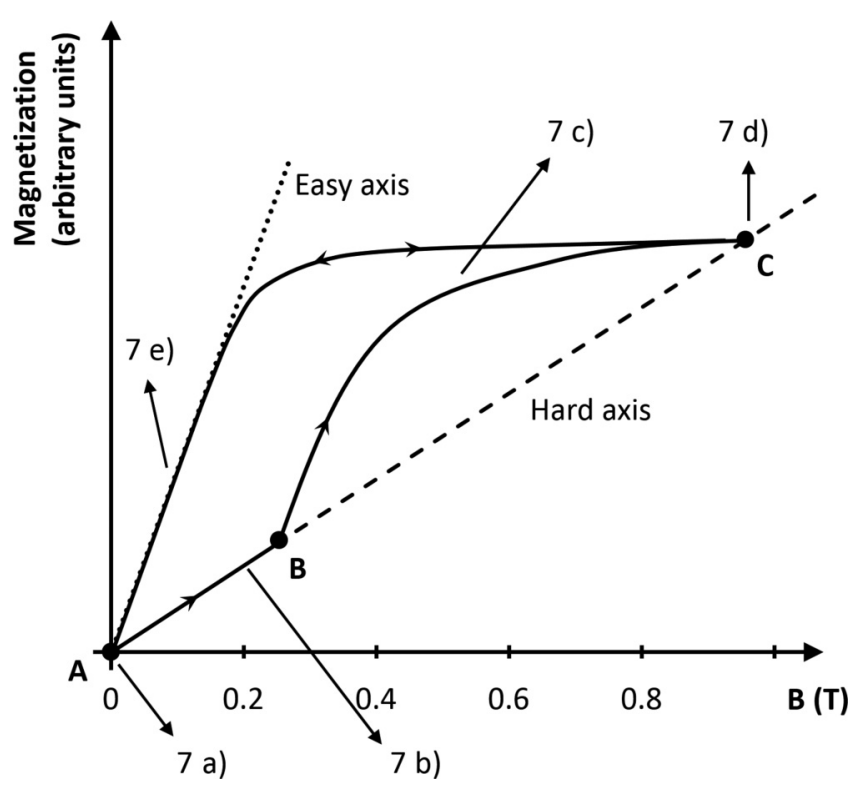

FIG. 8. Change of the magnetization, schematically during the first run (A-B-C path) and during the decrease (C-A path) or increase (A-C path) of the magnetic field after the first run. B corresponds to the start of detwinning. It can be seen that the A-B path is along the hard magnetization curve, while the low-field part of the A-C cycle lies along the easy magnetization curve (after Refs. [27] and [30]). The upper part of our experimental curves in Fig. 6 corresponds to this scheme.

$\varphi_{0}$, from the perpendicular position of the sample, i.e., let the $c$ axis not exactly perpendicular to the direction of the external field in Fig. 11(a). Then, switching up the external field, the domains with magnetization vector up [see again in Fig. 7(b)]

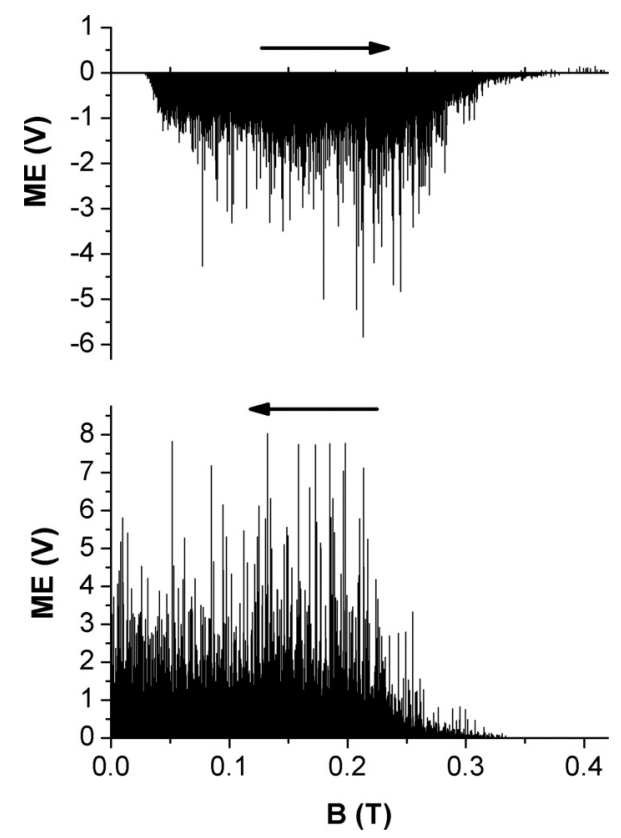

FIG. 9. Magnetic noise detected during increasing and decreasing the magnetic field between 0 and 0.3 T, i.e., stopping the increase of $\mathrm{B}$ before the deformation started (see Fig. 2) and decreasing it from this value.
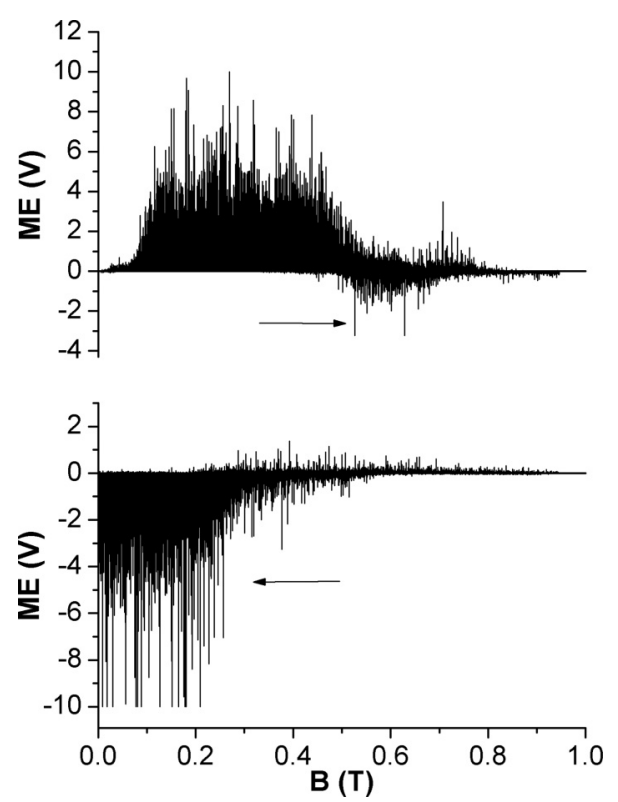

FIG. 10. Unidirectional Barkhausen signals, followed by twodirectional peaks if the sample was constrained (detwinning is not allowed). The arrows indicate the direction of the change of the field.

will be energetically more favorable and should increase their area, by domain wall shifts, leading to unidirectional signals. At the same time, during opposite domain rotations in the neighboring domains, until the $\Delta \varphi_{1}$ rotations are less than $\varphi_{0}$, the change of the vertical components will have the same sign: this can also create unidirectional signals in Fig. 11(b). For larger rotations in Fig. 11(c), the changes of the vertical components of the magnetizations will be opposite in the neighboring domains and signals of both directions should be detected. At the same time, the difference in the horizontal components will decrease leading to decrease of the driving force for domain wall shift. Furthermore, the changes in the vertical magnetization during rotations by less than $\varphi_{0}$, should be much less than the magnetization flips during the wall shifts.

Consequently, in accordance with Refs. [30,37], we attribute the low-field unidirectional BN to domain wall motions inside the initial variant [see Figs. 7(a) and 7(b)]. After some domain wall motions and simultaneous magnetization rotations, since the driving force for domain wall motion decreases, rotations with two-directional signals can dominate. Thus we attribute the two-directional signal package in Fig. 10 to domain rotations. We can conclude that at low-field values first domain wall motions appear and-if the formation of new twin variant(s) is prohibited the further magnetization process takes place by domain rotations. The importance of our conclusions is based on the possible separate detection of domain wall motions and rotations. In addition, the above arguments can also explain why only domain rotations were observed in Ref. [29] (if $\varphi_{0}$ is zero), or both domain wall shifts and rotations were detected here and summarized in the magnetomicrostructural model summarized in Sec. IV A.

The above explanation can be slightly modified if the initial state is not a completely single variant structure, having a small volume fraction of the original variant(s) (present in the state achieved by the compression), but the main conclusions should 


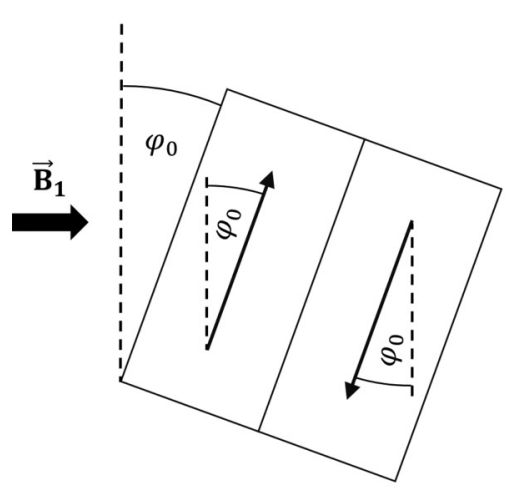

(a)

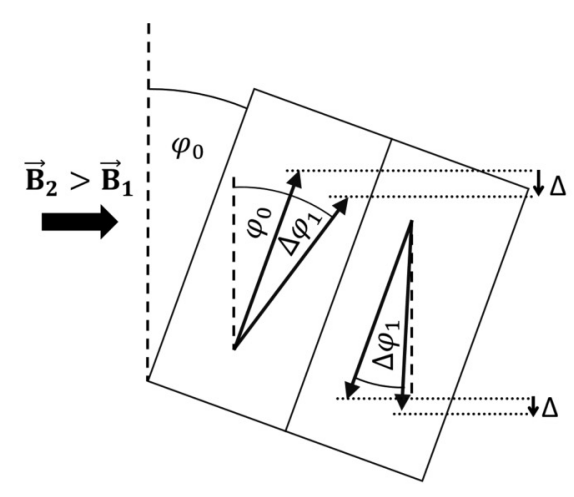

(b) $\Delta \varphi_{1}<\varphi_{0}$

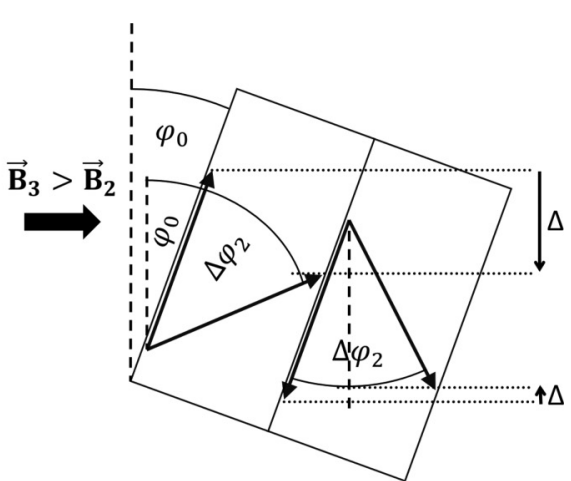

(c) $\Delta \varphi_{2}>\varphi_{0}$

FIG. 11. Changes of the vertical components of magnetizations of neighboring domains schematically, if there is a small misalignment, $\varphi_{0}$, from the exactly perpendicular position of domain magnetizations as compared to the direction of the field (see also the text).

remain the same. The weak unidirectional $\mathrm{ME}$ at low-field values in Fig. 4 is attributed to a classical type of Barkhausen noise during the domain rearrangement [see the transition from (e) to (d) in Fig. 7] during which intensive domain wall motions should take place. The fact that this unidirectional noise (with opposite direction) is about one order of magnitude weaker than the one shown in Fig. 2 is due to our measuring arrangements: the direction of the change of the magnetization is perpendicular to the long axis of the sample and thus only magnetization jumps in the large variant [see in Fig. 7(e)], slightly deviating from this direction, result in voltage peaks. Increasing the value of $B$ again, we observe, as it is shown in Fig. 5, a similar ME package (but with obviously opposite polarity) as shown in Fig. 4. This is just the reverse of the above Barkhausen noise due the change of magnetization by intermittent motion of domain walls [change from state (d) to (e) in Fig. 7]. A small contribution, related to the small shape change (see the small deformation in Fig. 4), can be present in this noise.

\section{Results on noises evoked by twin rearrangements}

In the first run (Fig. 2), the low-field BN signals diminish above about $B=0.20 \mathrm{~T}$ indicating that the main magnetic domain rearrangements by domain wall motions took place up to this field value. Above about $0.25 \mathrm{~T}$, weaker signals of both acoustic and magnetic origin appear and their activity is the strongest at around $0.8 \mathrm{~T}$. We attribute these peaks to the intermittent motion of the twin boundaries during the magnetic field induced superplastic deformation Figs. 7(c) and 7(d)] and to the simultaneous flips of magnetization during the twin boundary motion. It is indeed in accordance with the increasing elongation in the high field range (between 0.25 and $0.9 \mathrm{~T}$ ). Note that the switching value $B \cong 0.20 \mathrm{~T}$ is in a good agreement with the $0.145 \mathrm{mT}$ value observed in Ref. [29].

Decreasing the magnetic field (Fig. 4), weak AE noise is detected (note the about one order of magnitude difference in the vertical axes of Figs. 2 and 4) having the strongest activity at around $0.5 \mathrm{~T}$ at which the small deformation is located. Almost no magnetic signals can be seen around here, or they partly overlap with the tail of the unidirectional magnetic peaks at low-field values: see the noise package between 0 and $0.5 \mathrm{~T}$. Thus the weak AE activity (and also the weak ME) can be related to the small contraction at around $0.5 \mathrm{~T}$. This relative contraction (about $5 \times 10^{-3}$ ) cannot be the effect of magnetostriction since in this case the relative length change should be at least two orders of magnitude less. We rather attribute it to the following effect: the load due to the $0.9 \mathrm{~N}$ measuring force corresponds to a compressive stress of about $0.4 \mathrm{MPa}$. This is high enough to influence the final variant morphology even at the highest magnetic field. Thus at the end of the large deformation shown in Fig. 2 (at the largest magnetic field applied) the final state is probably not a completely single variant martensite structure [in contrast to Fig. 7(a)]. With decreasing magnetic field small twin rearrangement, driven by the compressive stress due to the measuring force, leading to the above contraction (which is about $9 \%$ of the relative deformation reached by the application of increasing magnetic field in the first run) took place at around $0.5 \mathrm{~T}$. This results in a very moderate $\mathrm{AE}$ and $\mathrm{ME}$ too.

In addition, there is a very minor $\mathrm{AE}$ and $\mathrm{ME}$ activity at high field values (between 0.8 and $0.9 \mathrm{~T}$ ) in Fig. 5 where a small elongation of the sample appeared. This is similar in magnitude to the contraction shown in Fig. 4, confirming that it is probably the reverse of the phenomenon discussed there with some hysteresis. Since the noise activities were very moderate during such a small length changes we could not carry out a statistical analysis and the corresponding exponents were not determined from noises detected here. Finally, we have observed, as it is expected, that after one magnetization cycle-if we restore the original multivariant structure by mechanical compression at $0 \mathrm{~T}$ magnetic field, a similar new cycle can be produced as shown in Figs. 2 and 4.

\section{Critical exponents}

\section{Critical exponents of magnetic noises due to magnetic domain rearrangements}

As we discussed in Introduction, the measured noises can be characterized by powerlike probability distribution functions, as given by Eq. (1). We have seen that different ranges can be observed in the magnetic emission: Barkhausen-type noises in the low-field range and magnetic noise due to twin boundary motion in the high-field range. In addition, the $\mathrm{BN}$ in the low-field range during the first run (the unidirectional 

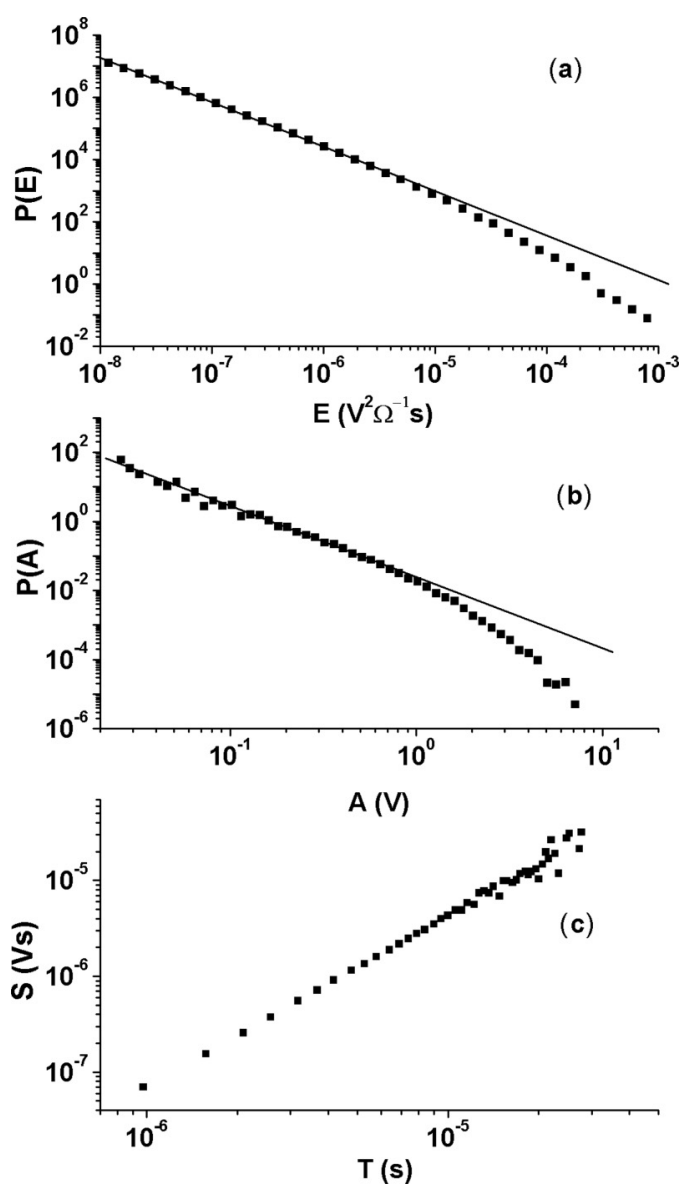

FIG. 12. Probability density functions of the peak energy, amplitude, and the scaling relation between the area $S$ and duration $T$ of magnetic emission signals in the low-field range [see Figs. 2 and 9, (a), (b), and c)], respectively. The straight lines indicate the fits with straight lines, i.e., neglecting the points in the cutoff range [see Eq. (1)].

package in Figs. 2 and 9) and the noise packages during the second run in Figs. 4 and 5 should belong to domain wall motions parallel and perpendicular to the external magnetic field, respectively [i.e., to magnetization along hard and easy directions: see Figs. 7(b) and 7(e)]. For the comparison with classical Barkhausen measurements, it is worth mentioning that the applied driving rate (corresponding to $4 \times 10^{-3} \mathrm{~T} \mathrm{~s}^{-1}$ ) corresponds to about $2 \mathrm{mHz}$, which is in the range of the recommended low values in order to avoid the overlapping of subsequent avalanches [31] generated by a triangular shape of the external field.

Figure 12 shows, as an illustration, the probability density functions of the energy and amplitude and the scaling relation between the area $S$ and the duration $T\left(S \sim T^{\gamma}\right)$ for the Barkhausen-type noises corresponding to the unidirectional signals shown in Figs. 2 and 9, belonging to magnetization along hard direction. We first calculated the above exponents for signals, shown in Figs. 2 and 9 separately, and they were the same for increasing and decreasing magnetic field. Thus the values shown in first row of Table I are the results of fitting to all peaks of these packages. The number of all events considered is shown in the last column of Table I.
For the determination of the critical exponents, after logarithmic binning, in accordance with Eq. (1), a threeparametrical nonlinear fitting of the probability distribution functions was made, using the Levenberg-Marquardt leastsquares method. The most sophisticated method for the estimation of the exponents is the maximum-likelihood method [38], which has only one fitting parameter. Unfortunately, this method cannot handle the exponential cutoff and if this value is relatively low [see, e.g., Fig. 12(b)], this fitting cannot be applied. Since our probability distribution functions were linear on a log-log scale over 3-4 orders of magnitude, the least-squares fitting gave the correct exponent with small fitting errors.

As we discussed earlier, the first unidirectional noise package shown in Fig. 10 corresponds to domain wall motions, while the second (two-directional) noise package most probably belongs to domain rotations when the magnetization is along the hard direction. Thus the critical exponents were calculated separately for these packages (by avoiding signals from the overlapping area). The second and third rows of Table I show the results of fits of the probability distributions of these two parts, respectively. As it can be seen, the exponents in the second row, as expected, are in a good agreement with those of the first row belonging to domain wall motions during magnetization along the hard direction too. Furthermore, they can be compared to those obtained in Ref. [11] (last row of Table I), from stress induced unidirectional magnetic signals. It can be seen that they are the same within the experimental errors, which is plausible since both unidirectional packages belong to a similar rearrangement of the magnetic domain structure with the only difference that in Ref. [11] the noise was evoked by elastic strain via magnetoelastic coupling, while here it was induced directly by the external magnetic field.

The third row of Table I contains the critical exponents, obtained from the two-directional noise packages belonging to domain rotations during magnetization along hard direction. These exponents are characteristically different from those belonging to domain wall motions.

The fourth row of Table I contains the values of the corresponding exponents evaluated from the BN packages shown in Figs. 4 and 5. According to Figs. 7(d), 7(e), and 8, this noise belongs to domain wall motions during magnetization along the easy magnetization direction. The fifth row in Table I, for comparison, contains the energy exponent determined in the multivariant martensitic state of a similar $\mathrm{Ni}_{2} \mathrm{MnGa}$ alloy with a bit different chemical composition $\left(\mathrm{Ni}_{55} \mathrm{Mn}_{20} \mathrm{Ga}_{25}\right)$ [39], measured also along the easy direction. The results in the fourth and fifth rows are in a very good agreement with each other, although the samples of Ref. [39] had a bit different composition than ours.

The marked differences between the magnitudes and the critical exponents of the Barkhausen signals excited along easy and hard magnetization directions (see Figs. 2, 4, 5, 9, and 10, as well as Table I) should be related to the specific magnetic domain structures, which are different in the two cases (see, e.g., Ref. [40]), and needs more attention in future experiments.

It is worth mentioning that, according to the literature, there exist two different universality classes of systems producing Barkhausen noises according to the range of interactions governing the domain wall motions: (i) long-range dipolar 
TABLE I. Critical exponents of ME and AE in the low-field range (see also the text).

\begin{tabular}{|c|c|c|c|c|}
\hline Magnetic field range & $\begin{array}{c}\text { ME energy } \\
\text { exponent }(\varepsilon)\end{array}$ & $\begin{array}{l}\text { ME amplitude } \\
\text { exponent }(\alpha)\end{array}$ & $\gamma$ & Number of events \\
\hline $\begin{array}{l}\text { Low-field }(0-0.25 \mathrm{~T}) \text {, increasing/decreasing field, } \\
\text { hard magnetization direction, unidirectional noise }\end{array}$ & $1.41 \pm 0.05$ & $2.0 \pm 0.05$ & $1.79 \pm 0.05$ & $1.7 \times 10^{5}$ \\
\hline Constrained sample, first run, increasing/decreasing field, & & & & \\
\hline $\begin{array}{l}\text { hard magnetization direction, unidirectional noise (low field), } \\
\text { domain wall motions }\end{array}$ & $1.3 \pm 0.1$ & $1.8 \pm 0.1$ & $1.76 \pm 0.05$ & $1.5 \times 10^{5}$ \\
\hline Constrained sample, first run, increasing/decreasing field, & & & & \\
\hline $\begin{array}{l}\text { hard magnetization direction, two-directional peaks, domain rotations } \\
\text { Low-field }(0-0.5 \mathrm{~T}) \text { second run, decreasing/increasing magnetic field, }\end{array}$ & $1.8 \pm 0.1$ & $2.6 \pm 0.1$ & $2.0 \pm 0.1$ & $1.5 \times 10^{5}$ \\
\hline easy magnetization direction, unidirectional noise, domain wall motions & $1.7 \pm 0.1$ & $2.3 \pm 0.1$ & $2.0 \pm 0.2$ & $2.8 \times 10^{5}$ \\
\hline $\begin{array}{l}\text { Exponents in martensitic state of } \mathrm{Ni}_{55} \mathrm{Mn}_{20} \mathrm{Ga}_{25} \\
\text { single crystal, easy magnetization direction [39] }\end{array}$ & $1.76 \pm 0.05$ & $2.37 \pm 0.1$ & - & $1.3 \times 10^{5}$ \\
\hline $\begin{array}{l}\text { Exponents of unipolar magnetic noise during deformation } \\
\text { induced magnetic emission [11] }\end{array}$ & $1.5 \pm 0.1$ & $2.0 \pm 0.1$ & - & $1.1 \times 10^{5}$ \\
\hline
\end{tabular}

interactions and (ii) short-range dipolar interactions [31-33]. These are characterized by different values of $\gamma$, exponent of peak area $\tau$, and durations $\alpha_{t} ; \tau=1.50 \pm 0.05, \alpha_{t}=2.0 \pm$ 0.2 , and $\gamma \equiv 2.0$, as well as $\tau=1.27 \pm 0.03, \alpha_{t}=1.5 \pm 0.2$, and $\gamma \equiv 1.77$, in classes $\mathrm{i}$ and $\mathrm{ii}$, respectively. Although the problem whether the different exponents obtained from domain wall motions during magnetization along easy and hard directions (and from domain rotations) belong to different universality classes, requires more detailed analysis (measurements and detailed evaluation of the values of $\tau$ and $\alpha_{t}$, too) and is beyond the framework of the present work, it is interesting that the values of $\gamma$ for the two magnetization directions are similar to the above values belonging to the different universality classes. Nevertheless, we evaluated the probability density function of peak durations for the domain wall motions along hard magnetization direction, for which we had the best statistics, since the number of events was the highest for this set. The result is shown in Fig. 13 and the exponent is $\alpha_{t} \cong 1.5$. This value and $\gamma$ obtained are in good agreement with the values belonging to class ii. Furthermore, the energy exponent, $\varepsilon=1.35 \pm 0.05$, determined in amorphous FINEMET-type ribbons (class ii) in Ref. [26] is also in good agreement with the value shown in the first row of Table I.

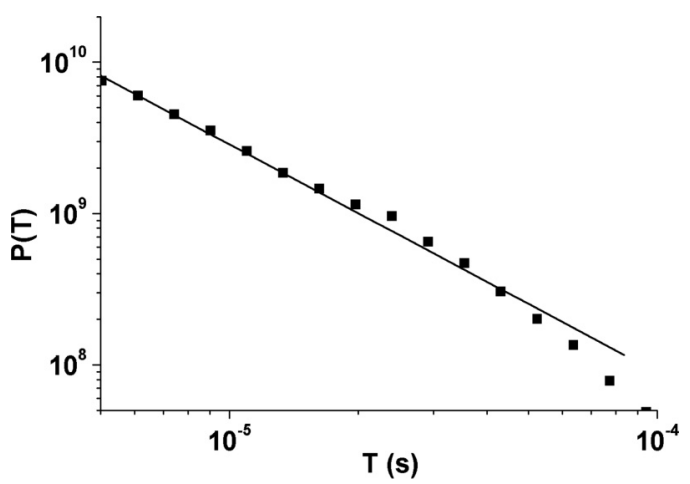

FIG. 13. Probability density function of peak durations for the Barkhausen noises obtained from magnetization along hard directions (see Figs. 2 and 9 and the first row in Table 1).

\section{Comparison of critical exponents of magnetic and acoustic noises obtained during intermittent variant rearrangements}

Figure 14 shows the energy probability density distributions for both the $\mathrm{ME}$ and $\mathrm{AE}$ in the high field range, i.e., during twin boundary motions, and the corresponding exponents are shown in the first row of Table II, together with the exponents of ME determined in Ref. [11] from deformation generated twin boundary motions in the same alloys at zero external magnetic field (second row). It can be seen that the corresponding critical exponents of ME and AE are very close to each other, which is in good accordance with the results of simultaneous measurement of $\mathrm{AE}$ and $\mathrm{ME}$ during austenite/martensite phase transformation in the same alloy [18].
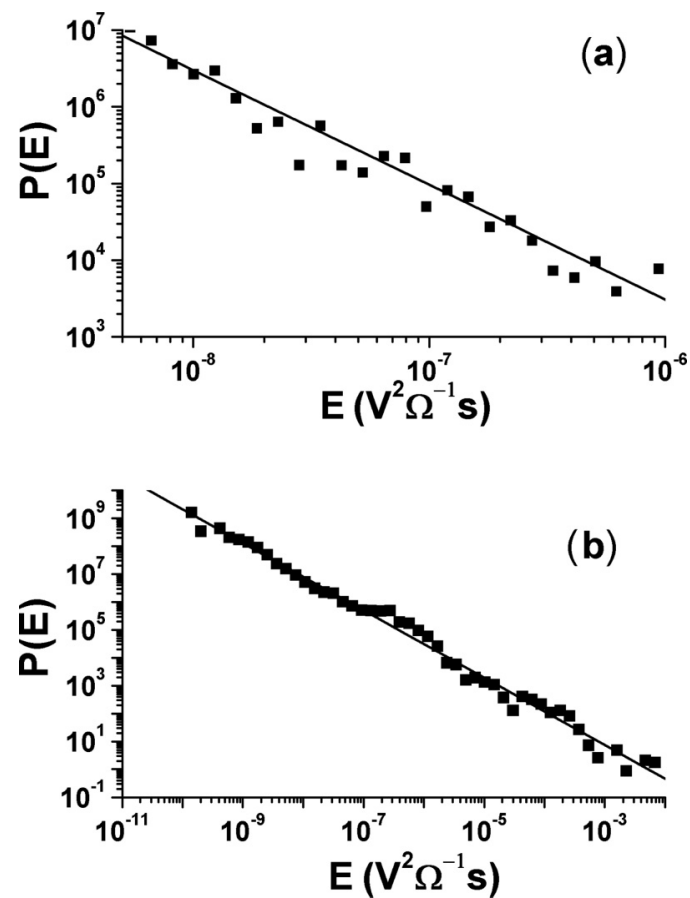

FIG. 14. Probability density functions of the peak energy for acoustic emission (a) and magnetic emission (b) signals in the high field range in Fig. 2. 
TABLE II. Critical exponents of energy and amplitude distribution functions for magnetic and acoustic emission noises due to intermittent variant rearrangements.

\begin{tabular}{|c|c|c|c|c|c|}
\hline Magnetic field range & $\begin{array}{c}\text { ME energy } \\
\text { exponent }(\varepsilon)\end{array}$ & $\begin{array}{l}\text { ME amplitude } \\
\text { exponent }(\alpha)\end{array}$ & $\begin{array}{c}\text { AE energy } \\
\text { exponent }(\varepsilon)\end{array}$ & $\begin{array}{l}\text { AE amplitude } \\
\text { exponent }(\alpha)\end{array}$ & Number of events \\
\hline High-field $(0.3-0.94 \mathrm{~T})$ first run & $1.4 \pm 0.1$ & $1.9 \pm 0.2$ & $1.5 \pm 0.1$ & $1.8 \pm 0.2$ & 6600 \\
\hline $\begin{array}{l}\text { Exponents obtained from cyclic deformation } \\
\text { in the same alloy at high magnetic field [11] }\end{array}$ & $1.6 \pm 0.1$ & $2.3 \pm 0.1$ & - & - & - \\
\hline
\end{tabular}

\section{E. Time correlations}

We have seen in Fig. 3 that a simple comparison of acoustic and magnetic emission pulses in a narrow field interval suggests strong correlations between these signals. For a deeper analysis of time correlations, we can follow the procedure proposed in Ref. [41] for the investigation of inhomogeneous temporal processes, consisting of highactivity bursty intervals alternating with long low-activity periods. This is based on the comparison of the experimentally determined $P\left(n ; t_{m}\right)$ functions with the $P_{\text {ind }}\left(n ; t_{m}\right)$ distributions $[18,21,41]$

$$
P_{\text {ind }}\left(n ; t_{m}\right)=a^{n-1}(1-a),
$$

belonging to sequence of $n$ successive independent events within a burst. Here, $t_{m}$ is the maximum time difference between subsequent events in a burst and $a\left(t_{m}\right)=\int_{0}^{t_{m}} P(t) d t$. Furthermore, $a^{n-1}$ and $(1-a)$ for independent events are the probability that $n-1$ events follow the first event with $t<t_{m}$ as well as the probability that for the subsequent event $t>t_{m}$ [18,41]. In Ref. [21], $t_{m}$ was typically about two orders of magnitude larger than the average waiting time, $\langle t\rangle=0.09 \mathrm{~s}$, between consecutive jerks. It can be seen from (2) that the asymptotic behavior is an exponential form: $P_{\text {ind }}\left(n ; t_{m}\right) \sim a^{n-1}$. On contrary, it was shown in Ref. [41] that in the case of correlated events the following scaling rule is fulfilled: $P\left(n ; t_{m}\right) \sim n^{-\beta}$ and the exponent $\beta$ is expected to have different values for different characteristic systems (classes), like mobile-call, email, or earthquake sequence.

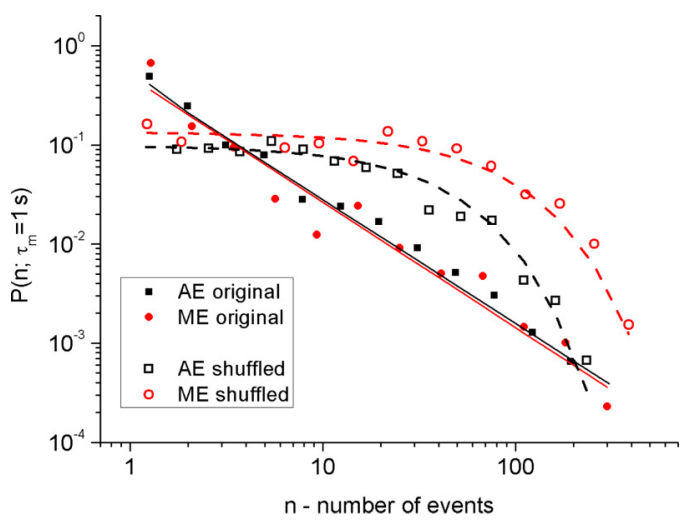

FIG. 15. $P\left(n ; t_{m}\right)$ distributions of a sequence of $n$ acoustic and magnetic events belonging to the same burst with $t<1 s$ and $P_{\text {ind }}\left(n ; t_{m}\right)$ distributions of randomized data sets (shuffled data). The solid lines show a power-law with $\beta=-1.25$ and the dashed lines show Eq. (2) (independent events) with $a=0.976$ for $\mathrm{AE}$ and $a=0.988$ for ME.
$P\left(n ; t_{m}\right)$ is quite robust against choosing the value of $t_{m}$ $[21,41]$ and a relatively small number of acoustic and magnetic events were detected $\left(10^{4}\right)$ during rearrangements of variants in the high-field range. Thus, in our case, $t_{m}$ was only about one order of magnitude larger than $\langle t\rangle, t_{m}=1 \mathrm{~s}$ to get more bursts and thereby better $P\left(n ; t_{m}\right)$ distributions.

Figure 15 shows the $P\left(n ; t_{m}\right)$ functions calculated from our experimental data for $t_{m}=1 \mathrm{~s}$. It can be seen that both functions (for $\mathrm{AE}$ and $\mathrm{ME}$ ) follow a power-law behavior, $P(n) \sim n^{\beta}$ with a common $\beta=-1.25$ exponent, indicating the time correlation between the events and the expected universal behavior. For a comparison, one can wipe out and ruin the time correlation with mixing the events of the original data sets. Figure 15 also shows the $P_{\text {ind }}\left(n ; t_{m}\right)$ distributions of the shuffled AE and ME data sets, which follow $P_{\text {ind }}\left(n ; t_{m}\right) \sim a^{n-1}$ asymptotic behavior with $a=0.976$ and $a=0.988$, respectively.

\section{CONCLUSIONS}

We have shown that jerky magnetic and acoustic noises can be evoked in martensitic single-crystalline $\mathrm{Ni}_{2} \mathrm{MnGa}$ samples by the application of external magnetic field. Applying the magnetic field along the hard magnetization direction of the sample with a single variant structure (made by compressive stress along the [100] direction), first, up to about $0.2 \mathrm{~T}$, unidirectional magnetic noise was detected and attributed to domain wall motions. At higher magnetic field, the detwinning process started (at about $0.2 \mathrm{~T}$ switching value), resulting in acoustic and two-directional magnetic signals, evoked by twin boundary motions.

Decreasing the magnetic field after the first run (after the formation of a new variant with easy axis parallel with the magnetic field) and cycling the sample in this state, again a unidirectional magnetic noise packet was observed (but weaker than in the first run) and attributed to domain wall motions during magnetization along the easy axis. If the sample was constrained during the first cycle (detwinning was not allowed) then the unidirectional magnetic noise was followed by a twodirectional noise packet, which was interpreted as the result of magnetic domain rotations.

Characteristic power-law exponents of amplitude and energy were determined for the above noises. It was found that the exponents that belong to domain wall motions agreed very well with those obtained in the same alloy during stress induced plastic deformation. Furthermore, characteristic exponents obtained when the magnetization is along the easy direction agree with those obtained from Barkhausen-noise measurements in a similar $\mathrm{Ni}_{2} \mathrm{MnGa}$ alloy with a bit different composition. 
The exponents were characteristically different for magnetizations along easy and hard magnetization directions and for domain wall motions and rotations. Time correlations within the magnetic as well as acoustic signals led to a power-law behavior, different from a distribution expected for uncorrelated events, with a common scaled power function for both types of signals.

\section{ACKNOWLEDGMENTS}

This work was supported by the Hungarian National Research, Development and Innovation Office in the framework of the GINOP-2.3.2-15-2016-00041 Project, cofinanced by the European Regional Development Fund, related to the Europe 2020 Strategy.
[1] O. Heczko, N. Scheerbaum, and O. Gutfleisch, in Nanoscale Magnetic Materials and Applications, edited by J. P. Liu, E. Fullerton, O. Gutfleisch, and D. Sellmyer (Springer US, New York, 2009), pp. 399-439.

[2] A. Sozinov, A. A. Likhachev, N. Lanska, and K. Ullakko, Appl. Phys. Lett. 80, 1746 (2002).

[3] B. Krevet, V. Pinneker, and M. Kohl, Smart Mater. Struct. 21, 094013 (2012).

[4] K. Ullakko, L. Wendell, A. Smith, P. Müllner, and G. Hampikian, Smart Mater. Struct. 21, 115020 (2012).

[5] O. Heczko, Mater. Sci. Technol. 30, 1559 (2014).

[6] P. Ranzieri, M. Campanini, S. Fabbrici, L. Nasi, F. Casoli, R. Cabassi, E. Buffagni, V. Grillo, C. Magén, F. Celegato et al., Adv. Mater. 27, 4760 (2015).

[7] A. Turabi, H. Karaca, H. Tobe, B. Basaran, Y. Aydogdu, and Y. Chumlyakov, Scr. Mater. 111, 110 (2016).

[8] O. Heczko, P. Cejpek, J. Drahokoupil, and V. Holý, Acta Mater. 115, 250 (2016).

[9] L. Straka, V. Novak, M. Landa, and O. Heczko, Mat. Sci. Eng. A374, 263 (2004).

[10] L. Daróczi, S. Gyöngyösi, L. Z. Tóth, S. Szabó, and D. L. Beke, Appl. Phys. Lett. 106, 041908 (2015).

[11] L. Daróczi, S. Gyöngyösi, L. Z. Tóth, and D. L. Beke, Scr. Mater. 114, 161 (2016).

[12] G. Bertotti, Hysteresis in Magnetism for Physicists, Material Scientists, and Engineers (Academi Press, San Diego, 1998), pp. 255-259.

[13] J. P. Sethna, K. A. Dahmen, and C. R. Myers, Nature (London) 410, 242 (2001).

[14] S. Papanikolaou, F. Bohn, R. L. Sommer, G. Durin, S. Zapperi, and J. P. Sethna, Nat. Phys. 7, 316 (2011).

[15] L. Laurson, X. Illa, S. Santucci, K. T. Tallakstad, K. J. Måløy, and M. J. Alava, Nat. Commun. 4, 2927 (2013).

[16] E. K. Salje and K. A. Dahmen, Annu. Rev. Condens. Matter Phys. 5, 233 (2014).

[17] A. Planes, L. Mañosa, and E. Vives, J. Alloys Compd. 577(Suppl. 1), S699 (2013).

[18] L. Z. Tóth, L. Daróczi, S. Szabó, and D. L. Beke, Phys. Rev. B 93, 144108 (2016).

[19] L. Z. Tóth, S. Szabó, L. Daróczi, and D. L. Beke, Phys. Rev. B 90, 224103 (2014).

[20] R. Niemann, J. Kopecek, O. Heczko, J. Romberg, L. Schultz, S. Fähler, E. Vives, L. Mañosa, and A. Planes, Phys. Rev. B 89, 214118 (2014).
[21] Z. Kovács, M. Ezzeldien, K. Máthis, P. Ispánovity, F. Chmelík, and J. Lendvai, Acta Mater. 70, 113 (2014).

[22] I. Shashkov, M. Lebyodkin, and T. Lebedkina, Acta Mater. 60, 6842 (2012).

[23] L. Carrillo and J. Ortín, Phys. Rev. B 56, 11508 (1997).

[24] Y. Ge, O. Heczko, O. Söderberg, and V. Lindroos, J. Appl. Phys. 96, 2159 (2004).

[25] M. R. Sullivan and H. D. Chopra, Phys. Rev. B 70, 094427 (2004).

[26] Y. Ge, O. Heczko, O. Söderberg, and S.-P. Hannula, Scr. Mater. 54, 2155 (2006).

[27] A. Likhachev and K. Ullakko, Phys. Lett. A 275, 142 (2000).

[28] O. Heczko, J. Magn. Magn. Mater. 290, 787 (2005).

[29] Y. Lai, N. Scheerbaum, D. Hinz, O. Gutfleisch, R. Schäfer, L. Schultz, and J. McCord, Appl. Phys. Lett. 90, 192504 (2007).

[30] H. Karaca, I. Karaman, B. Basaran, Y. I. Chumlyakov, and H. Maier, Acta Mater. 54, 233 (2006).

[31] G. Durin and S. Zapperi, The Science of Hysteresis (Elsevier, Amsterdam, 2006), p. 181.

[32] G. Durin and S. Zapperi, Phys. Rev. Lett. 84, 4705 (2000).

[33] F. Bohn, M. A. Corrêa, A. D. C. Viegas, S. Papanikolaou, G. Durin, and R. L. Sommer, Phys. Rev. E 88, 032811 (2013).

[34] Z. Balogh, L. Daróczi, L. Harasztosi, D. L. Beke, T. A. Lograsso, and D. L. Schlagel, Mater. Trans. 47, 631 (2006).

[35] G. Eszenyi, S. Szabó, L. Harasztosi, F. Zámborszky, J. Nyéki, Z. Erdélyi, and D. L. Beke, J. Mater. Res. 24, 130 (2009).

[36] G. Bertotti, F. Fiorillo, and M. Sassi, J. Magn. Magn. Mater. 23, 136 (1981).

[37] H. Kato and K. Sasaki, Scr. Mater. 48, 31 (2003).

[38] A. Clauset, C. R. Shalizi, and M. E. Newman, SIAM Rev. 51, 661 (2009).

[39] L. Daróczi, A. Bükki-Deme, Z. Balogh, L. Harasztosi, Z. Erdélyi, D. Beke, T. A. Lograsso, and D. L. Schlagel, in Proceedings of the International Conference on Shape Memory and Superelastic Technologies, edited by S. Miyazaki (ASM International, USA, 2008), p. 607.

[40] V. Kopecký, L. Fekete, O. Perevertov, and O. Heczko, AIP Adv. 6, 056208 (2016).

[41] M. Karsai, K. Kaski, A.-L. Barabási, and J. Kertész, Sci. Rep. 2, 397 (2012). 